\title{
Adenitis cervical en pediatría: 5 años de caracterización clínica y paraclínica
}

\author{
Cervical adenitis in pediatrics: 5 years of clinical \\ and paraclinical characterization
}

\author{
Sebastián Medina-Rivera, ${ }^{*, \ddagger}$ Kelly Márquez-Herrera, ${ }^{\ddagger}$, Karen Yuliana Ramírez-Iriarte, ${ }^{\ddagger}$ Carolina Ramos-Ramos ${ }^{\ddagger}$ \\ * Pediatra. \\ ₹ Departamento de Pediatría. Facultad de Medicina. \\ § Pediatra. Infectóloga Pediatra. Universidad Nacional Autónoma de México. \\ Universidad Nacional de Colombia. Bogotá, Colombia.
}

\section{RESUMEN}

Introducción: Las linfadenopatías cervicales son una manifestación frecuente en la infancia y son un motivo de consulta común. Es importante reconocer aquéllas que se asocian a un proceso infeccioso local, para determinar la etiología y realizar un tratamiento antimicrobiano adecuado. Objetivo: Identificar las características clínicas, microbiológicas e histopatológicas, asociadas con cuadros de adenitis cervical en pacientes de 0-18 años, atendidos en un hospital pediátrico de cuarto nivel. Material y métodos: Estudio de tipo observacional analítico de corte transversal. Se revisaron las historias clínicas de pacientes de 0-18 años con adenitis cervical, atendidos en la Fundación Hospital de la Misericordia de Bogotá, entre el 1 de enero de 2013 y el 31 de diciembre de 2018. Resultados: Se incluyeron 447 pacientes, $53.7 \%$ fueron de sexo masculino, con una mediana de edad de cuatro años. El dolor local, la induración y la fiebre se encontraron en $70,61.7$ y $56 \%$ de los casos, respectivamente. Se realizaron pruebas serológicas en 59 pacientes con seroprevalencia de $67.7 \%$ para citomegalovirus y $63.7 \%$ para Epstein Barr, $10 \%$ de los pacientes requirió drenaje quirúrgico; se identificó $S$. aureus como el germen más frecuente en $41 \%$ de los aislamientos, con sensibilidad a oxacilina en $68.4 \%$ de los casos, $79.6 \%$ de los pacientes recibió tratamiento antibiótico, siendo cefalexina (35.8\%) y clindamicina (24.6\%) los más utilizados. Conclusiones: Existe gran subregistro de las características clínicas de las adenitis y un amplio uso de antibiótico sin indicación. Los agentes causales coinciden con los reportados en la literatura. Se desaconseja el uso rutinario de antibiótico empírico, dada la baja frecuencia de progresión a lesión supurativa.

Palabras clave: Adenitis cervical, pediatría, ganglio, infección cervical.

\section{ABSTRACT}

Introduction: Cervical lymphadenopathy is a frequent manifestation in childhood and is a common reason for consultation. It is important to recognize those that are associated with a local infectious process, to determine the etiology and carry out an adequate antimicrobial treatment. Objective: To identify the clinical, microbiological and histopathological characteristics associated with cervical adenitis symptoms presented in patients aged 0-18 years, treated in a fourth level pediatric hospital. Material and methods: Cross-sectional analytical observational study. The medical records of patients aged 0-18 years with cervical adenitis, treated at the Fundación Hospital de la Misericordia in Bogotá, between January 1, 2013 and December 31, 2018 were reviewed. Results: 447 patients were included, $53.7 \%$ were male, with a median age of four years. Local pain, induration, and fever were found in 70, 61.7, and $56 \%$, respectively. Serological tests were performed in 59 patients with seroprevalence of $67.7 \%$ for cytomegalovirus and $63.7 \%$ for Epstein Barr. $10 \%$ of the patients received surgical drainage; $S$. aureus was identified as the most frequent germ in $41 \%$ of the isolates, susceptible to oxacillin in $68.4 \% .79 .6 \%$ of the patients received antibiotic treatment, with cephalexin (35.8\%) and clindamycin (24.6\%) being the most used. Conclusions: There is a large underreporting of the clinical characteristics of adenitis and a wide use of antibiotics without indication. The causal agents coincide with those reported in the literature. The routine use of empirical antibiotics is discouraged, given the low frequency of progression to suppurative injury.

Keywords: Cervical adenitis, pediatrics, ganglia, cervical infection.

Citar como: Medina-Rivera S, Márquez-Herrera K, Ramírez-Iriarte KY, Ramos-Ramos C. Adenitis cervical en pediatría: 5 años de caracterización clínica y paraclínica. Rev Latin Infect Pediatr. 2021; 34 (4): 187-192. https://dx.doi.org/10.35366/102969

Recibido: 09-11-2020. Aceptado: 02-12-2020.

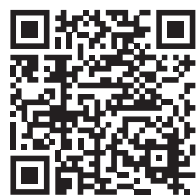




\section{INTRODUCCIÓN}

Las linfadenopatías cervicales son una de las patologías más frecuentes en la edad pediátrica y tienen un espectro variado de etiologías, lo cual hace necesaria la realización de un estudio regional que busque establecer las diferentes causas, plantear abordajes diagnósticos y tratamientos pertinentes y oportunos. ${ }^{1,2}$

El término adenitis hace referencia a la presencia de signos inflamatorios locales y regionales asociados. ${ }^{1}$ La etiología viral suele ser la más frecuente a nivel global; en países endémicos cobran importancia las adenitis de tipo tuberculoso, y en países desarrollados son más frecuentes las debidas a bacterias típicas y micobacterias no tuberculosas, Staphylococcus aureus y Streptococcus pyogenes son los microorganismos aislados con mayor frecuencia. ${ }^{3-5}$

De acuerdo con las características de la adenitis o de los síntomas acompañantes, deben realizarse algunas pruebas específicas; tal es el caso de la prueba de tuberculina si se sospecha tuberculosis, la radiografía de tórax en aquellos pacientes que presenten adenitis de curso crónico o que generalice y las pruebas serológicas específicas para citomegalovirus (CMV), virus de Epstein Barr (EBV), brucelosis, sífilis, virus de inmunodeficiencia humana $(\mathrm{VIH})$ y toxoplasmosis. ${ }^{6}$ Dentro del estudio de las adenitis, existen abordajes invasivos y no invasivos, las imágenes son de especial ayuda para la determinación de abscesos, mientras que los drenajes y biopsias son útiles para el diagnóstico etiológico final. ${ }^{7,8}$

Existe un desconocimiento respecto a la incidencia, las características de la población pediátrica afectada y los microorganismos causales de adenitis en nuestro medio, debido probablemente al subregistro y a la ausencia de publicaciones referentes a este tema, lo cual hace difícil una comprensión de las características de la enfermedad e impide la adecuada prescripción empírica de los antibióticos. El objetivo de este artículo es identificar las características clínicas, microbiológicas e histopatológicas, asociadas con cuadros de adenitis cervical en pacientes de 0-18 años, atendidos en un hospital pediátrico de cuarto nivel.

\section{MATERIAL Y MÉTODOS}

Estudio de tipo observacional descriptivo de corte transversal retrospectivo. Previa autorización por parte del Comité de Ética de la Fundación Hospital de la Misericordia, se realizó la búsqueda y revisión de los datos en las historias clínicas electrónicas de pacientes entre cero y 18 años con diagnóstico de adenitis cervical, atendidos en los servicios de urgencias, hospitalización y consulta externa de la institución, entre el 1 de enero de 2013 y el 31 de diciembre de 2018.

Se obtuvo un total de 1,773 historias clínicas de pacientes con adenitis cervical, de las cuales fueron incluidas en el estudio 447 historias que contaron con datos completos. Debido a la falta de estudios que describan de forma clara la prevalencia de adenitis cervical en pediatría, se realizaron los cálculos de muestra en el programa EPIDAT versión 4.1, teniendo en cuenta los siguientes parámetros: prevalencia conocida $50 \%$ (la máxima esperada para un estudio transversal en el cual no es clara la prevalencia teórica), confianza de $95 \%$ y precisión de $4 \%$, dando como resultado una muestra necesaria de 257 participantes, esta muestra representa la mínima cantidad de casos necesarios para llevar a cabo la investigación.

La base de datos se registró en Excel versión 2013 y se analizó en el programa estadístico SPSS versión 21. Para las variables cualitativas se determinaron frecuencias absolutas y relativas y en el caso de las variables cuantitativas se utilizaron medidas de tendencia central, medidas de dispersión o medidas de posición (empleando media y desviación estándar cuando la variable numérica sigue la distribución normal o mediana, rango intercuartílico y percentiles en el caso contrario).

\section{RESULTADOS}

De los 447 casos analizados en esta cohorte, el sexo más frecuente fue el masculino con $53.7 \%$, la mediana de edad fue de cuatro años. Las manifestaciones clínicas más frecuentes fueron dolor local en $76.7 \%$, induración de la lesión en $61.7 \%$ y fiebre en $56.8 \%$. Por el contrario, signos como el eritema, la adherencia a planos profundos, la pérdida de peso y la supuración crónica, estuvieron presentes sólo en el $21.7,12.1,1.1$ y $1.3 \%$, respectivamente (Tabla 1).

Sólo se dispuso del reporte de hemograma en $69.5 \%$ de los casos. En el estudio predominó la presencia de leucocitosis con media de 13,930 células $/ \mathrm{mm}^{3}$, los valores de hemoglobina y plaquetas se mantuvieron en la mediana de la normalidad (Tabla 2). 
Tabla 1: Manifestaciones clínicas de los pacientes

con adenitis cervical atendidos en Fundación HOMl en los años 2013-2018 ( $N=447)$.

\begin{tabular}{lc}
\hline Características clínicas & Resultados, $\mathrm{n}(\%)$ \\
\hline Locales & \\
\hline Localización & \\
$\quad$ Cervical & $443(99.1)$ \\
$\quad$ Supraclavicular & $4(0.9)$ \\
Tamaño & Mediana: $2 \mathrm{~cm} 1-5 \mathrm{~cm}$ \\
& Moda: $2 \mathrm{~cm}$ \\
Tiempo de evolución & Mediana: 3 días $1-150$ días \\
& Moda: 1 día \\
Dolor & $343(76.7)$ \\
Induración & $276(61.7)$ \\
Eritema & $97(21.7)$ \\
Adherencia a planos profundos & $54(12.1)$ \\
Supuración & $6(1.3)$ \\
\hline Sistémicas & \\
\hline Fiebre & $254(56.8)$ \\
Duración de la fiebre & Moda: 1 día \\
Pérdida de peso & $5(1.1)$ \\
&
\end{tabular}

La mayoría de pacientes (86.8\%) no contaba con pruebas serológicas en búsqueda de etiología de la adenitis. Aquéllos que contaban con el examen mostraron altas tasas de seroprevalencia (IgG positiva) para CMV (67.7\%) y para EBV $(63.7 \%)$. Los resultados de las serologías encontradas se muestran en la Tabla 3. La prueba de tuberculina fue solicitada en $0.7 \%$, siendo negativa en los tres casos en que se realizó.

Respecto a estudios de imagen, se realizó radiografía de tórax en 14\%, siendo normal en 62 de los 63 pacientes. Se realizó ecografía abdominal en $12.3 \%$ $(n=55)$, en la mayoría de los casos fue normal $(80 \%$ $[n=44]) ;$ sin embargo, $2 \%$ de los pacientes $(n=9)$ presentó esplenomegalia.

Se realizó drenaje quirúrgico en $9.6 \%$ de los pacientes, el germen aislado con mayor frecuencia fue $S$. aureus en $4 \%, 4.4 \%(n=20)$ de los pacientes fue sometido a biopsia de ganglio, encontrando como hallazgos histológicos más frecuentes hiperplasia folicular reactiva (ocho pacientes), cambios inflamatorios (cinco pacientes) y granulomatosis crónica con necrosis (cinco pacientes). Se realizó inmunohistoquímica en seis pacientes, encontrando como hallazgo más frecuente cambios compatibles con infección por Barto- nella henselae; la reacción en cadena de polimerasa (PCR) para micobacterias fue solicitada sólo en $2 \%$ de los casos, sin resultados positivos (Tabla 4).

De acuerdo con los reportes de cultivo, inmunohistoquímica y PCR para micobacterias según el número de muestras realizadas, Staphylococcus aureus fue la bacteria más frecuentemente aislada en cultivo con $41.8 \%$, Bartonella henselae fue la más común por hallazgos de inmunohistoquímica con $16.6 \%$, en la muestra analizada no se presentó ningún caso de PCR positiva para micobacterias (Tabla 5). Respecto a los patrones de sensibilidad de los dos microorganismos aislados en cultivo, $68.4 \%$ de $S$. aureus aislado fue sensible a oxacilina, el único aislamiento de $S$. pyogenes mostró sensibilidad a penicilina. Se administró tratamiento antibiótico en $79.6 \%$ de los casos, la cefalexina fue el antibiótico prescrito con mayor frecuencia en $35.8 \%$, seguido por clindamicina en $24.6 \%$, el tiempo de duración de tratamiento tuvo una mediana de siete días.

\section{DISCUSIÓN}

La población más afectada dentro del estudio fueron los preescolares, con una mediana de cuatro años, datos que concuerdan con los trabajos de Gosche y Leung, ${ }^{9,10}$ quienes describen este grupo etario como el más comprometido por adenitis de origen bacteriano. Dentro de los hallazgos más comunes de adenitis se encontraron: la fiebre en $56 \%$ de la población estudiada con duración media de un día, aumento de tamaño con una mediana de $2 \mathrm{~cm}$, induración en $61.7 \%$ y dolor en $70 \%$; mientras que el eritema y la supuración aparecen en menos de la mitad de la muestra, con 21.7 y $1.3 \%$, respectivamente. Otros hallazgos asociados a malignidad como la adherencia a planos profundos (12\%) y

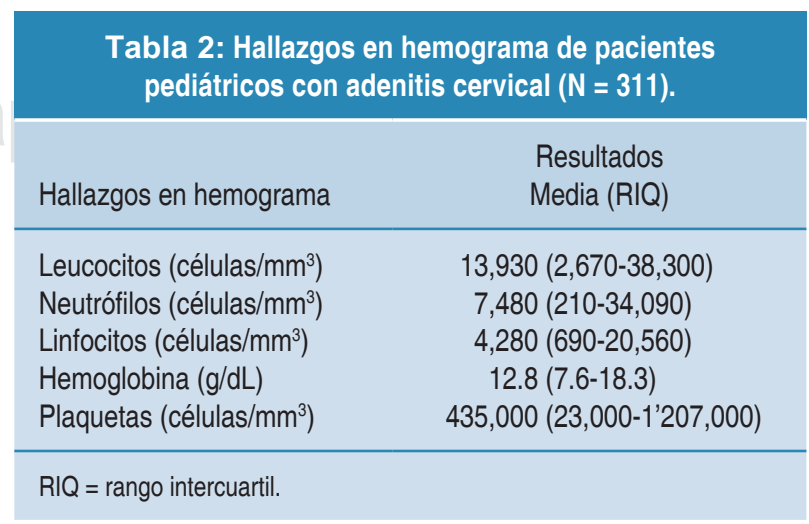


Tabla 3: Resultados de las serologías de pacientes con adenitis cervical según cantidad de serologías realizadas.

\begin{tabular}{|c|c|c|c|c|}
\hline \multirow[b]{2}{*}{ Serología solicitada } & \multirow[b]{2}{*}{$\begin{array}{c}\text { Cantidad de serologías } \\
\text { realizadas }\end{array}$} & \multicolumn{2}{|c|}{ Serologías } & \multirow[b]{2}{*}{$\begin{array}{l}\text { Zona gri } \\
\text { n (\%) }\end{array}$} \\
\hline & & $\begin{array}{l}\text { Positivas } \\
\text { n (\%) }\end{array}$ & $\begin{array}{l}\text { Negativas } \\
n(\%)\end{array}$ & \\
\hline \multicolumn{5}{|l|}{ Toxoplasma } \\
\hline $\lg M$ & 54 & $0(0)$ & $54(100)$ & $0(0)$ \\
\hline $\lg G$ & 44 & $4(9)$ & $40(91)$ & $0(0)$ \\
\hline \multicolumn{5}{|l|}{ Bartonella henselae } \\
\hline $\lg M$ & 5 & $1(20)$ & $4(80)$ & $0(0)$ \\
\hline $\lg G$ & 5 & $3(60)$ & $2(40)$ & $0(0)$ \\
\hline \multicolumn{5}{|l|}{ Citomegalovirus } \\
\hline $\lg M$ & 68 & $12(17.6)$ & $52(79.4)$ & $4(3)$ \\
\hline $\lg G$ & 59 & $40(67.7)$ & 19 (32.3) & $0(0)$ \\
\hline \multicolumn{5}{|l|}{ Epstein Barr } \\
\hline $\lg M$ & 66 & $3(4.5)$ & 61 (92.4) & $2(3.1)$ \\
\hline $\lg G$ & 58 & $37(63.7)$ & $21(36.3)$ & $0(0)$ \\
\hline VIH anticuerpos & 14 & $2(14.3)$ & $12(85.7)$ & $0(0)$ \\
\hline
\end{tabular}

pérdida de peso (1.1\%) fueron infrecuentes en la población estudiada.

La etiología del cuadro fue evaluada a partir de pruebas serológicas para los microorganismos descritos con mayor frecuencia en la literatura como agentes causales de adenitis; sin embargo, las historias clínicas revelaron un porcentaje muy alto de pacientes sin pruebas serológicas (86.8\%), por lo cual en ocasiones no se logró establecer un diagnóstico etiológico. Se encontró infección aguda por citomegalovirus en 2.7\% $(n=12)$, Epstein Barr en $0.7 \%(n=3)$ y $\mathrm{VIH}$ en $0.2 \%(n=2)$, datos que en orden de frecuencia son similares a los descritos en los trabajos de Leung. ${ }^{9,10}$ Otros microorganismos reportados con menor frecuencia en la literatura tuvieron porcentajes igualmente bajos en el presente estudio, tales como Bartonella henselae en $0.7 \%$ (n $=3$ ) y Toxoplasma sin evidencia de casos.

De la muestra total, 9.6\% $(n=43)$ fue llevado a drenaje quirúrgico, de éstos $41 \%(n=18)$ tuvieron aislamiento en cultivo de $S$. aureus y $2 \%(n=1)$ de S. pyogenes, sin otros aislamientos considerados como patógenos (los dos gérmenes adicionales fueron considerados contaminantes); estos dos microorganismos están ampliamente referenciados como las etiologías bacterianas más comunes. ${ }^{1,5,9,10}$ La etiología bacteriana se describe de forma más frecuente en países de primer mundo, tal como lo reporta Inman. ${ }^{3}$
No se evidenciaron cambios significativos en los conteos celulares del hemograma. La radiografía de tórax fue realizada en $14 \%$, siendo normal en 62 de 63 casos, con un único paciente con hallazgo de adenopatías intratorácicas, hallazgos que no se pueden comparar por ausencia de información en la literatura. La prueba de tuberculina fue solicitada en $0.7 \%$, siendo negativa en los tres casos en que se solicitó. La ecografía abdominal se realizó en 12.3\% (n $=55)$, en la mayoría de los casos fue normal $(80 \%$ [ $n=$ 44]); sin embargo, $2 \%$ de los pacientes $(n=9)$ presentó esplenomegalia. Nuevamente tenemos limitación en la comparación de datos por no existir estudios que caractericen estos hallazgos en pacientes con adenitis cervicales, aunque existen datos en pacientes con adenomegalias cervicales que presentan patologías con compromiso sistémico como las oncológicas. ${ }^{7}$

Cuatro punto cuatro por ciento $(n=20)$ fue llevado a biopsia, encontrando como hallazgos más frecuentes la hiperplasia folicular reactiva en $38 \%(n=8)$, cambios reactivos en $25 \%(n=5)$ y granulomatosis crónica con necrosis en $25 \%(n=5)$ de las muestras. Sólo una muestra demostró presencia de necrosis caseificante, hallazgo patognomónico de tuberculosis. Se encontraron estudios de inmunohistoquímica en $29.5 \%(n=6)$ de las biopsias analizadas $(1.3 \%$ del total de pacientes) con hallazgo de hongos no tipificables en $4.5 \%(n=1)$, micobacterias en $9 \%(n=$ 2) y $15.4 \%(n=3)$ fueron positivas para $B$. henselae, 
Rev Latin Infect Pediatr. 2021; 34 (4): 187-192

datos que no son comparables, ya que las caracterizaciones que se encuentran en la literatura se han hecho sobre adenomegalias no necesariamente asociadas a signos de adenitis.

Se administró tratamiento antibiótico en $79.6 \%$ $(n=356)$, la cefalexina fue el antibiótico prescrito con mayor frecuencia en $38.5 \%(n=160)$, seguido por clindamicina en $24.6 \%(n=110)$ y ampicilina sulbactam en $1.3 \%(n=6)$, con una duración media de tratamiento de siete días, lo que coincide con la

\begin{tabular}{|c|c|}
\hline Variable & Resultado, n (\%) \\
\hline \multirow{6}{*}{$\begin{array}{l}\text { Cultivo del drenaje } \\
\text { quirúrgico }(\mathrm{N}=43)\end{array}$} & S. aureus: 18 (4.0) \\
\hline & S. pyogenes: $1(0.2)$ \\
\hline & Enterococcus durans: 1 (0.2) \\
\hline & Staphylococcus \\
\hline & pseudintermedius: 1 (0.2) \\
\hline & Negativo: 22 (4.9) \\
\hline \multirow{6}{*}{$\begin{array}{l}\text { Histopatología } \\
(\mathrm{N}=20)\end{array}$} & Hiperplasia folicular reactiva: 8 (1.8) \\
\hline & Cambios inflamatorios: 5 (1.1) \\
\hline & Granulomatosis crónica \\
\hline & con necrosis: 5 (1.1) \\
\hline & Necrosis caseificante: 1 (0.2) \\
\hline & Pilomatricoma: 1 (0.2) \\
\hline \multirow{5}{*}{$\begin{array}{l}\text { Hallazgo en } \\
\text { inmunohistoquímica } \\
(\mathrm{N}=6)\end{array}$} & Bacterias: $0(0)$ \\
\hline & Hongos: 1 (0.2) \\
\hline & Micobacterias: $2(0.4)$ \\
\hline & Toxoplasmosis: $0(0)$ \\
\hline & Bartonella henselae: $3(0.7)$ \\
\hline PCR para & Positivo: 0 (0) \\
\hline micobacterias $(\mathrm{N}=8)$ & Negativo: 8 (2.0) \\
\hline
\end{tabular}

información aportada por Rajasekaran ${ }^{5}$ respecto a los tratamientos antibióticos de primera línea para adenitis bacterianas; sin embargo, no se encontró información sobre la duración usual de estos tratamientos diferente a las recomendaciones de Gosche que sugiere siete días totales.

Cabe anotar que durante la revisión de las historias clínicas, se encontró una ausencia significativa de datos del examen físico y de estudios adicionales que permitieran concluir una etiología específica de la adenitis cervical, al tratarse de un estudio de tipo retrospectivo, ésta fue una limitación importante para el análisis de los resultados y debe ser tenida en cuenta en el momento de extrapolar los mismos a otro tipo de poblaciones.

Además, la realización de un estudio prospectivo planteaba limitaciones metodológicas importantes debido al desconocimiento de la prevalencia de adenitis cervical en nuestro medio, es por esto que se decidió realizar el estudio de manera retrospectiva, sabiendo de antemano que los datos que encontraríamos plantearían un segundo reto, por la limitante del registro incompleto de los datos en la historia clínica.

\section{CONCLUSIONES}

En el análisis de los datos fue evidente un subregistro de las características clínicas en la historia médica de atención inicial, con un número importante de pacientes sin estudios de primera línea para abordaje etiológico de las adenitis.

Es importante reconocer que las características de la adenitis cervical no necesariamente se tienen que presentar completas y que el cuadro clínico encon-

\begin{tabular}{lcc}
\multicolumn{2}{c}{ Tabla 5: Reporte de cultivo, inmunohistoquímica y PCR para micobacterias según número } \\
de muestras realizadas en pacientes pediátricos con adenitis cervical.
\end{tabular}

PCR = reacción en cadena de polimerasa; SAMS = Staphylococcus aureus meticilino sensible; SAMR = Staphylococcus aureus meticilino resistente. 
trado en nuestro estudio corresponde a un síndrome febril agudo de corta duración y con hallazgos locales como su principal marcador, especialmente el aumento de tamaño, la induración y el dolor.

La supuración fue un hallazgo poco frecuente, lo que guarda relación con las etiologías posibles, siendo los virus el grupo más grande, como en la mayoría de las patologías infecciosas de la edad pediátrica. En aquellos casos con progresión a enfermedad supurativa, los agentes etiológicos concuerdan con los descritos en la literatura mundial, siendo $S$. aureus el germen más frecuente, seguido por $S$. pyogenes.

De los 18 aislamientos de $S$. aureus en cultivo obtenido a partir de drenaje quirúrgico de la adenitis, $68.4 \%$ fueron sensibles a oxacilina, por lo que, sin ser el objetivo del estudio, se aconseja como primera línea de tratamiento antibiótico para pacientes pediátricos con adenitis cervical el uso de cefalexina u oxacilina según corresponda la vía de administración, con cobertura para $S$. aureus meticilino sensible y $S$. pyogenes, al ser éstos los dos agentes etiológicos más frecuentes tanto en el presente estudio como en la literatura.

Esperábamos encontrar un mayor número de casos con diagnóstico de infección por micobacterias, dada la prevalencia intermedia de enfermedad tuberculosa en nuestro país (17.7 casos/100,000 habitantes): $;{ }^{11}$ sin embargo, los resultados mostraron un número muy bajo de estos aislamientos, que puede estar relacionado con la búsqueda de patología de localización cervical como manifestación única.

Debe tenerse en cuenta que la biopsia de ganglio es un estudio invasivo con indicaciones específicas, por esta misma razón, en el presente estudio la frecuencia de biopsia y estudios de inmunohistoquímica fue muy baja, con menos de $5 \%$ de los pacientes, lo que limita la búsqueda de otros gérmenes frecuentes en la literatura como la Bartonella sp. o Toxoplasma sp., que podrían llevar a cambios terapéuticos dentro del manejo.

Desde los hallazgos patentes en el análisis del presente estudio, se desaconseja el uso de terapia antibiótica empírica para el tratamiento de la adenitis cervical no supurativa, ya que la mayoría de los cuadros son de etiología viral o se autolimitan y, de ser necesario su uso por hallazgos clínicos que sugieran infección bacteriana o por no existir una auto- rresolución del cuadro, se recomienda la búsqueda de aislamiento microbiológico para reducir tiempos de tratamiento antibiótico y lograr una terapéutica dirigida al germen aislado, buscando también disminuir la presión de selección sobre los antibióticos y el creciente problema de resistencia bacteriana.

\section{AGRADECIMIENTOS}

A la Fundación Hospital de la Misericordia por el acceso a los datos para la realización del trabajo.

\section{REFERENCIAS}

1. Gosche JR, Vick L. Acute, subacute, and chronic cervical lymphadenitis in children. Semin Pediatr Surg. 2006; 15 (2): 99-106.

2. Niedzielska G, Kotowski M, Niedzielski A, Dybiec E, Wieczorek P. Cervical lymphadenopathy in childrenIncidence and diagnostic management. Int $\mathrm{J}$ Pediatr Otorhinolaryngol. 2007; 71 (1): 51-56.

3. Inman JC, Rowe M, Ghostine M, Fleck T. Pediatric neck abscesses-Changing organisms and empiric therapies. Laryngoscope. 2008; 118 (12): 2111-2114.

4. Barton LL. Principles and practice of pediatric infectious diseases. JAMA. 2003; 289 (22): 3016-3017.

5. Rajasekaran K, Krakovitz P. Enlarged neck lymph nodes in children. Pediatr Clin N am. 2013; 60: 923-936.

6. Weinstock MS, Patel NA, Smith LP. Pediatric cervical lymphadenopathy. Pediatr Rev [Internet]. 2018; 39 (9): 433443. Available in: http://pedsinreview.aappublications.org/ content/39/9/433

7. Kelly CS, Kelly J. Lymphadenopathy in children. Pediatr Clin North Am [Internet]. 1998; 45 (4): 875-888. Available in: http://www.ncbi.nlm.nih.gov/pubmed/9728192

8. Nolder AR. Paediatric cervical lymphadenopathy: When to biopsy? Curr Opin Otolaryngol Head Neck Surg. 2013; 21 (6): 567-570.

9. Leung AKC, Davies HD. Cervical lymphadenitis: etiology, diagnosis, and management. Curr Infect Dis Rep. 2009; 11 (3): 183-189

10. Leung AK, Robson WLM. Childhood cervical lymphadenopathy. J Pediatr Heal Care [Internet]. 2004; 18 (1): 3-7. Available in: http://linkinghub.elsevier.com/retrieve/ pii/S0891524503002128

11. Instituto Nacional De Salud. Boletín Epidemiológico. Una mirada hacia el comportamiento de la tuberculosis en Colombia, 2018 Semana epidemiológicas 1-36.

Financiamiento: Ninguno.

Conflicto de intereses: Ninguno.

Correspondencia:

Sebastián Medina-Rivera

E-mail: scmedinar@unal.edu.co 